\title{
Optimization design method of multimedia technology in large-scale broadcasting and television project
}

\author{
Zhou Xing ${ }^{1}$, Chuping $\mathrm{Wu}^{2}$ \\ 1. School of Humanities and Art, Nanchang Institute of Technology, Nanchang Anhui 330099, China \\ 2. School of Humanities and Art, Nanchang Institute of Technology, Nanchang Anhui 330099, China
}

Keywords: image compression algorithm; broadcasting and television project; multimedia

\begin{abstract}
. in the field of multimedia technology, image compression technology is at the core position, which directly affect the quality of the large-scale broadcasting and television project. In the traditional wavelet multimedia video image compression technology, the shortcomings of wavelet coefficient characteristics and wavelet coefficient quantization based on frequency characteristic, which severely damage the multimedia compressed image. Aiming at these problems, a compression algorithm of multimedia image is proposed based on improved wavelet domain sub vector, using improved LBG vector quantization method based on quantization compensation error to preprocess multimedia image, designing model from point to line, the low frequency part is selected from two dimension wavelet domain as the sub vectors, error compensation code method is adopted for the details of the sub vector in the wavelet domain to smooth multimedia image noise, two level wavelet transformation is utilized for $\mathrm{N}$ code book to extract the low-frequency coefficients, through the arithmetic coding to achieve multi-source information multimedia image compression and decoding, so as to recover recognition results. The experimental results show that, compared with the traditional wavelet transform image compression algorithm, the improved multimedia image compression algorithm proposed in the paper improves the quality of multimedia compressed image.
\end{abstract}

\section{Introduction}

The compression technology of multimedia image in broadcasting and television project is the key for images [1,2]. The traditional multimedia image compression method large-scale broadcasting and television project is based on image statistics and visual characteristics, these methods cannot meet the requirements of multimedia image transmission and processing. With further research, in recent years, the multimedia image compression method based on wavelet theory, machine learning theory and chaos theory appears in broadcasting and television project $[3,4]$. Wavelet division is suitable for the principle of human vision and has multiresolution, which is a multimedia image processing method with high compression speed and high compression accuracy for large-scale broadcasting and television project currently, however, for the multimedia image with rich texture information, wavelet can only hold the information in horizontal and vertical direction, can not be a good representation of texture and edge information $[5,6]$.

In large-scale broadcasting and television project, multimedia image compression field has received wide attention, many mature algorithms have appeared such as EZW coding [7,8], SPIHT coding and so on. Shapiro employed zero tree to handle wavelet coefficients of the image, and made use of the interband correlation and the innerband correlation effectively, obtained higher coding efficiency. The theory of Shannon information theory showed that, for memoryless sources, vector quantization is always better than the scalar quantization.

This paper presents a compression algorithm of multimedia image based on improved wavelet domain sub vector, compared with the traditional wavelet transform image compression algorithm, the improved multimedia image compression algorithm proposed in the paper improves the quality of multimedia compressed image. 


\section{Multimedia image compression and LBG vector quantization preprocessing}

\section{1 the process of Multimedia image compression and coding ideology}

(1) in the original large-scale broadcasting and television project, multimedia images have a lot of redundant information, the redundant information have correlation in the space, vision and structure, detail layer of grey levels of adjacent pixels are often highly correlated, for the effective implementation of multimedia image coding and transmission, data compression is required;

(2) Through multimedia image compression in large-scale broadcasting and television project to remove the redundant information, and keep important information of multimedia image, in order to meet the sensitivity of the eyes for multimedia image brightness and resolution requirements for multimedia image color, image restored by multimedia image compression under high compression ratio is more conducive to improving the quality of multimedia image.

The multimedia image in large-scale broadcasting and television project is regarded as random vector model of a patch of the point to the line, wavelet transform is adopted to divide images into a plurality of detail layers, and the vector quantization analysis is conducted to realize multimedia image compression.

The nature of multimedia image compression of large-scale broadcasting and television project is the image vector quantization coding, coding idea is as follows: assuming multimedia image is divided into $M$ vectors with the length $\mathrm{L}$, a multimedia image is viewed as a string of data, each segment have L data, $M$ vectors in the low frequency part of multimedia image are grouped as $N$, the code book size is $\mathrm{N}$, with the corresponding vector quantization coding method to obtain the generated code $\boldsymbol{W}_{j}(\mathrm{j}=1,2, \ldots, \mathrm{N})$, the representative in $\mathrm{j}$-th group is $Y_{j}, \quad j=0,1, \cdots N-1$, multimedia images in large-scale broadcasting and television project are compressed.

2.2 multimedia image pre processing based on $L B G$ vector quantization

According to the process of multimedia image compression and coding in large-scale broadcasting and television project, the first step to conduct low distortion and lossless compression for multimedia image is preprocessing multimedia image with LBG vector quantization method, the idea of multimedia image coding LBG algorithm is: for a set of multimedia image sequence, the structural information feature decomposition method is utilized to find the quantitative center, and produce an initial code $\hat{A}_{0}$, the adjacent pixels is grouped in $\hat{A}_{0}$, and the center vector matching method is employed to correct vector symbol, the specific steps are described below:

(1) initialize: the quantized code $N$ of multimedia image in large-scale broadcasting and television project is given, the independent threshold is $\varepsilon$, training samples of multimedia images is $\left\{x_{j}\right\}, j=0,1, \cdots, m-1$, multimedia image is divided into two parts, the approximation and detail, the available codebook training samples of $N$ level is:

$$
\hat{A}_{0}=\left\{y_{i}\right\}, i=1,2, \cdots, N
$$

Supposing $n=0, D_{-1}=\infty$.

(2) given $\hat{A}_{n}=\left\{y_{i}\right\}, i=1,2, \cdots, N$, using wavelet decomposition to remove the high frequency part of multimedia image $\left\{x_{j}\right\}, j=0,1, \cdots, m-1$, quantization error of lossless image concerning $\hat{A}_{n}$ is

$$
P\left(\hat{A}_{n}\right)=\left\{s_{i}\right\}, i=1,2, \cdots, N
$$

Wherein $s_{i}=\left\{x_{j}: d\left(x_{j}, y_{i}\right) \leq d\left(x_{j}, y_{1}\right)\right\}$ represents a record number in the code book at corresponding position, for noise $l=0,1, \cdots, N$ of multimedia image in large-scale broadcasting and television project, the total average distortion is calculated:

$$
D_{n}=D\left(\left\{P\left(\hat{A}_{n}, \hat{A}_{n}\right)\right\}\right)=\frac{1}{m} \sum_{j=0}^{m-1} \min _{y \in \hat{A}_{n}} d\left(x_{j}, y\right)
$$

(3) if $\left(D_{n-1}-D_{n}\right) / D_{n} \leq \varepsilon$, stop; $\hat{A}_{n}$ is final codebook of multimedia image vector quantization output; otherwise, continue.

(4) does not change the space division, so that $m$ data becomes the center of each group of $M$ data vector correction, bits required by new pixel multimedia image is obtained:

$$
\hat{x}\left(P\left(\hat{A}_{n}\right)\right)=\left\{\hat{x}\left(s_{j}\right)\right\}, j=1,2, \cdots, N
$$


(5) the total error of the new codebook for current vector space partition is minimum, i.e.

$$
\hat{x}\left(s_{j}\right)=\frac{1}{\left\|s_{j}\right\|} \sum_{x_{i} \in s_{j}} x_{i}
$$

Among them, $\left\|s_{j}\right\|$ is the number of sample vectors in $s_{j}$.

(6) $\hat{A}_{n+1}=\hat{x}\left(s_{j}\right)$,setting $n=m+1$, go to step (2) .

\section{3 improvement of multimedia image preprocessing $L B G$ vector quantization algorithm}

In this paper, the quantization error compensation method is adopted to optimize LBG vector quantization algorithm. The method described as: if center vector of multimedia image is $R, k$, let $N=2^{k R / 2}$, multimedia image detailed signal components of wavelet decomposition is $\left\{x_{j}\right\} j=0,1, \cdots, m-1$; through $N$ level of detailed layer LBG algorithm, the codebook $\hat{A}^{(0)}$ is obtained, low frequency coefficient parameters $y_{j}{ }^{(0)}$ is solved in quantization process, $y_{j}{ }^{(0)} \in \hat{A}^{(0)}$. Error correction coding, get:

$$
\xi_{j}=x_{j}-y_{j}{ }^{(0)}
$$

The quantization error sequence $\left\{\xi_{j}\right\}$ is accessed, $j=0,1, \cdots, m-1$; quantization error is utilized to compensate for quantized multimedia image, so as to acquire codebook $\hat{A}^{(1)}$, end cycle, achieve improved LBG image vector quantization method, as the pretreatment results of multimedia image compression.

2.4 Realization and processing of improved Multimedia image compression

On the basis of preprocessing the multimedia image, point to line model is designed based on the triangle cosine theorem.

The low frequency part in wavelet domain is selected as sub vectors, the codebook decomposed by LBG vector quantization in point to line model has $\mathrm{N}$ levels, $\boldsymbol{C}_{L}{ }^{2}$ combination, look for shortest distance vector combination in the model, error compensation coding is $\boldsymbol{S}_{k}(\mathrm{k}=1,2, \ldots, \mathrm{M})$, using two-dimensional wavelet decomposition method, a series of different high resolution sub image are obtained, as a subspace of wavelet domain, wecodemat wavelet function is selected from the two-dimensional wavelet function, , expression is:

$$
p_{i, j}(A)= \begin{cases}\frac{w_{i, j}}{w_{i}} & \text { if } i \neq j \text { and } e_{i, j} \in A \\ 0 & \text { if } i \neq j \text { and } e_{i, j} \notin A \\ 1-\frac{\sum_{j: e_{i, j} \in A} w_{i, j}}{w_{i}} & \text { if } i=j\end{cases}
$$

According to the wavelet sub vector characteristics to remove space information redundancy, vision redundancy and structure redundancy of multimedia image and of visual spatial information, after that, the following information is obtained:

$$
\begin{gathered}
m_{p q}=\sum_{m=1}^{M} \sum_{n=1}^{N} x^{p} y^{q} f(x, y) \\
\mu_{p q}=\sum_{m=1}^{M} \sum_{n=1}^{N}(x-\dot{x})^{p}(y-\dot{y})^{q} f(x, y) \\
n_{p q}=\frac{\mu_{p q}}{\left(\mu_{00}\right)^{\gamma}}
\end{gathered}
$$

\section{Experimental analysis}

In order to test the proposed multimedia image compression method for large-scale broadcasting and television project, a lot of experiments were conducted. All experiments were performed in Matlab6.5 environment. The multimedia original image in large-scale broadcasting and television project is grayscale image in standard TIF format 0.

The statistical results of the wavelet coefficient vectors and lowest resolution sub test coefficients of multimedia image were listed, the average energy of vector is defined as 
$E=\frac{1}{N} \sum_{i=1}^{N} \sum_{k=1}^{K} v_{i, k}{ }^{2}$, where $N$ is the number of vectors, $K$ is the dimension of vectors. The improved algorithm was adopted for multimedia image compression processing of large-scale broadcasting and television project, the obtained results can be described by the following table:

Table 1 the statistics of performance of improved algorithm

\begin{tabular}{|c|c|c|}
\hline Image & Compression ratio / times & peak signal to noise ratio $/ \mathrm{dB}$ \\
\hline Image a & 89.67 & 22.98 \\
\hline Image b & 88.98 & 23.98 \\
\hline Image c & 90.56 & 27.84 \\
\hline Image d & 89.22 & 26.98 \\
\hline Image e & 90.13 & 22.86 \\
\hline Image f & 89.44 & 21.99 \\
\hline Image g & 92.11 & 26.98 \\
\hline Image h & 87.56 & 22.76 \\
\hline Image i & 83.86 & 25.98 \\
\hline Image j & 89.71 & 26.81 \\
\hline
\end{tabular}

According to the table it can be known that, with the proposed algorithm to compress multimedia image in large-scale broadcasting and television project, multimedia technology is optimized, which meets the actual demand of large-scale broadcasting and television project.

\section{Conclusion}

This paper presents a compression algorithm of multimedia image based on improved wavelet domain sub vector, using improved LBG vector quantization method based on quantization compensation error to preprocess multimedia image, designing model from point to line, the low frequency part is selected from two dimension wavelet domain as the sub vectors, error compensation code method is adopted for the details of the sub vector in the wavelet domain to smooth multimedia image noise, two level wavelet transformation is utilized for $\mathrm{N}$ code book to extract the low-frequency coefficients, through the arithmetic coding to achieve multi-source information multimedia image compression and decoding, so as to recover recognition results. The experimental results show that, compared with the traditional wavelet transform image compression algorithm, the improved multimedia image compression algorithm proposed in the paper improves the quality of multimedia compressed image.

\section{Reference}

[1] Wang Zhiwen. Multimedia image compression technology [J]. Journal of Jixi University: edition, 2005, (1): 72-73.

[2] Zhang Zhigang. Analysis of multimedia image compression algorithm [J]. Computer programming skills and maintenance, 2013, (22): 69-70.

[3] Zhu Xiaobo. Multimedia communication and image compression technology [J]. Airport construction, 2008, (4): 32-33.

[4] Ye Wenjun, Zheng Yang, Geng Xinmin. Compression algorithm in heterogeneous distributed parallel fractal image based on genetic algorithm [J]. Computer applications, 2006, 26 (4): 793-797.

[5] Huang Changchun, Xu Shuyan, Hu Jun. Research of remote sensing image compression algorithm based on Singular value decomposition [J]. Computer simulation, 2011, 28 (8): 226-228.

[6] Li Dongmei. Visual information description and compression technology in multimedia application [C]. Modern TV technology, 2001:31-46. 
[7] Luo Hui, Liu Juefu. Image data compression coding in multimedia communication [J]. Journal of East China Jiaotong University, 2003, 20 (4): 62-65.

[8] Yin Lujuan, Yuan Jinsha, Guo Zengwei. Research of image compression technology [J]. China multimedia communications, 2009, (7): 47-49. 